\title{
Cystic fibrosis as a bowel cancer syndrome and the potential role of CK2
}

\author{
Anil Mehta
}

Received: 20 May 2008/ Accepted: 29 May 2008/Published online: 5 July 2008

(C) Springer Science+Business Media, LLC. 2008

\begin{abstract}
Chloride is critical in creating differential $\mathrm{pH}$ values inside various organelles (Golgi for example) by linking ATP hydrolysis to trans-bilayer proton movement. This proton-ATPase drives anions such as chloride through unrelated channels in the endosomal/organellar bilayer thus loading $\mathrm{HCl}$ into different lipid-encased cellular compartments. Critically, intraorganellar $\mathrm{pH}$ (and ion channel content/activities) differs during different phases of the cell cycle. The cystic fibrosis (CF) chloride channel protein CFTR is a member of the ABC family (ABCC7) and resides in many endosomal membranes trafficking to the epithelial surface and back again. Recently, it has become clear that human $\mathrm{CF}$ has an unusually high incidence of cancer in the bowel with correspondingly elevated gut epithelial proliferation rates observed in $\mathrm{CF}$ mice. In this review, emphasis is placed on CK2 \& CF because CK2 controls not only proliferation but also four different members of the ABC superfamily including the multi-drug resistance protein P-glycoprotein and CFTR itself. In addition, CK2 also regulates a critical cancer-relevant and CFTR-regulated cation channel $(\mathrm{ENaC})$ that mediates the cellular accumulation of sodium ions within epithelia such as the colon and lung. Not only are ENaC and CFTR both abnormal in CF cells, but ENaC also 'carries' CK2 to the cell membrane in oocytes, only provided its two target phosphosites are intact. CK2 may be a critical regulator of cell proliferation in conjunction with regulation of ion channels such as CFTR, other ABC members and the cation channel ENaC. The emerging idea is that CFTR may
\end{abstract}

\footnotetext{
A. Mehta $(\bowtie)$

Division of Maternal and Child Health Sciences, Ninewells

Hospital, University of Dundee, Dundee DD1 9SY, UK

e-mail: a.mehta@dundee.ac.uk
}

control membrane-CK2 as much as membrane-CK2 controls CFTR.

Keywords Apical · Ion channel - Sodium - Transport

\begin{tabular}{|c|c|c|}
\hline \multicolumn{3}{|c|}{ Abbreviations } \\
\hline CFTR & $\begin{array}{l}\text { Cystic fibrosis transmembrane } \\
\text { regulator }\end{array}$ & conductance \\
\hline $\mathrm{ABC}$ & ATP-binding cassette & \\
\hline PD & Potential difference & \\
\hline $\mathrm{ENaC}$ & Epithelial sodium channel & \\
\hline NBD & Nucleotide binding domain & \\
\hline
\end{tabular}

\section{Introduction}

Epithelia balance two conflicting roles, namely, keeping the organism's outside-world out and the inside-world in, whilst maintaining the facility to secrete and absorb across the epithelial barrier. This epithelial layer may be a single sheet of cells or multi-stacked (interlinked by hemi-desmosomes to a stem cell layer) but in all cases, the distal membrane of the outermost cell faces the milieu surrounding the organism. It is commonly found that electrodes placed on an epithelial surface, when connected to another in the blood, register a few millivolts difference on a voltmeter. This review links that finding to a protein kinase involved in cancer biology. The essential protein kinase (CK2, formerly known as casein kinase 2) has been implicated in multiple steps in neoplasia. As a first step towards linking this finding to an ion channel, emerging data are discussed suggesting that $\mathrm{CK} 2$ can control different members of the ATP-binding cassette (ABC) superfamily and one of their interacting cation channels. 


\section{Materials and methods}

Epithelia are polarised, but in two completely different 'vectorial' (directional) contexts.

- Firstly, the lipid and protein composition of the cell membrane that faces the outer world (the apical membrane, typically 25 square microns or about $6 \%$ of the total cell surface area) differs from the majority inward-facing membrane in contact with the intercellular matrix (basolateral membrane). Differences in lipid composition may be relevant for the relationship between CK2 function and endocytosis in such membranes (discussed below).

- Further, the surface area of both apical and basolateral membranes in each epithelial cell (in a fully grown organism) is held relatively constant by balancing lipid and protein synthesis (that underpins new membrane arrival from the Golgi-generated budding vesicles) with the reverse process of membrane retrieval due to endocytosis-critically, ion channels are involved in such vesicle cycling and vesicle $\mathrm{pH}$. Any change in cell area will modulate cell volume, of necessity.

- Epithelial cell number is also held constant by balancing stem cell turnover in the lower, blood facing layers with subsequent differentiation and directional cell migration towards the surface of the epithelium. This balances programmed cell death where once again, as described below, different ion channels are critically important. Cancers occur in such epithelia when the controllers of cellular renewal and cell death become disordered in a sequential process. Thus a polyp may be considered as a 'buckled' epithelium.

- The second type of polarity is electrical and arises from the differential membrane potentials generated across these apical and basolateral membranes; a potential arises from a net charge difference across a space creating a force felt by all charged particles, should opposite charges not be able to move towards each other to cancel the force, a potential difference (PD) is said to exist. Each apical and basolateral bilayer maintains a PD that is set at its own resting level by virtue of its protein content. This PD is created by the free diffusion of charged potassium (but not other) ions through highly ion-selective channel proteins which themselves differ in each membrane. Both apical and basolateral PDs are 'negative inside' at rest and set by the (in to out) leakage of positively charged potassium ions whose concentration inside the cells is 30 -fold in excess of that found outside (140 and $4.5 \mathrm{mM}$, respectively). Potassium is topped up by the sodium potassium ATPase which can consume $40 \%$ of cell energy even at rest. Thus highly regulated $\mathrm{K}^{+}$exit creates a relative inner negativity as
$\mathrm{K}^{+}$diffuses down its concentration gradient through any transiently open $\mathrm{K}^{+}$-selective pore. Multiple such pores exist, all regulated differently. Discussion focuses in this paper on changing the expression of a type of $\mathrm{K}^{+}$channel for example to a calcium-activated or ATPgated $\mathrm{K}^{+}$channel which has been proposed as a controller of the cell cycle.

- The different PD-magnitude across two membranes in the same epithelial cell can occur simultaneously because of electrically resistant protein moieties at the Zona Occludens (ZO). This apical-apical insulating junction between two epithelial cells is often called a tight junction and 'spot-welds' the most lateral ends of adjacent apical membranes to one other. In reality, such junctions are variably leaky and plastic but this is beyond the scope of the article but nevertheless, this leads to the idea of a resistance to flow of current across an epithelium either across individual cells or between cells as a trans-epithelium PD relative to the blood.

- To bridge the interface between cancer biology and electrophysiology, a key technical limitation has to be considered that affects interpretation of the data. Protein serum factors may be excluded from normal (but not cancer-breached) epithelia by compartments such as the basal lamina that normally separates the basal cells in an epithelium from the underlying capillaries/blood stream. The technical difficulty is that serum factors are present in cell culture but not in vivo. The result is that epithelial cells often show different ion channel characteristics when compared in serum to the balanced salt solutions favoured by electrophysiologists during their ion channel studies. For example, a rapidly turning over ion channel may disappear when placed in balanced salts.

\section{Results}

Cancer and ion channels: a new frontier

Electrophysiology and cancer cell biology do not always make good bed fellows. To paraphrase one prominent cancer researcher, "whenever we came across an ion channel as a cancer gene, we ignored it, not really knowing what question to ask let alone, answer'. Two reviews were published in 2005 that bridge that gap [1, 2]. For example, Kunzelmann [2], who began as an electrophysiologist but has widened his research into tumour biology [3], states that you cannot apoptose without shrinking cell volume. To an electrophysiologist this means you need at least a potassium channel with a high throughput of ions per second (high conductance, the inverse of resistance) to 
drive cell-shrinkage but typical value of $40 \mathrm{mM}$ intracellular chloride must co-exit by its own anion-selective channels to maintain charge neutrality. Water follows. The cell shrinks. This process, when defective, has been recently linked to cancer cell drug resistance [4]. Further, Kunzelmann points out [2] that the menu of available $\mathrm{K}^{+}$channels differs in different parts of the cell cycle. For example, when cell volume needs to be stable ('BK' channels found mainly in $\mathrm{S}$ phase are present) or when membrane voltage needs to be in a special range, intermediate conductance (IC) $\mathrm{K}^{+}$channels in G1 phase create a bigger negative-inside potential to 'attract in' ionised calcium; cytosolic calcium is characteristically elevated at this time from the low nanomolar to the micromolar range; outside cells and inside some organelles, calcium is millimolar. Further still, to exit $\mathrm{G} 1$, another type of $\mathrm{K}^{+}$-channel may be needed (a $\mathrm{K}^{+}$-ATP gated channel). Overall this suggests that co-ordinated, energy-regulated, sequential expression of different channels at different times is somehow linked to cell volume, cell death and cell division $[1,2]$. In summary, the working hypothesis is that any cell will have basal homeostatic roles fulfilled by multiple ion channels, but will need to induce/regulate a different set of channels or proliferation/senescence will not occur in normal sequence [3]. It follows that a cell that fails to express the right type of channels for apoptosis may be unable to perform this action.

\section{Such $\mathrm{K}^{+}$-channels do not act alone}

They coordinate their activities with chloride/anion movement to drive changes in cellular/organellar ion composition and hence cell/organellar volume (often called regulatory cell volume increase, RVI or decrease RVD). Chloride is an abundant mobile intracellular anion, unlike a (negatively charged) cell cytosol protein, say. This volume recovery process goes awry in CF cells as found by the elegant work of Valverde and co-workers many years ago [5] and is now linked to cancer [4]. The presence of cell membrane proteins generates $90 \%$ of trans-bilayer water movement which otherwise has a very low permeability across bilayer lipids [6]. Chloride permeability is low in G0 and rises through G1 to $\mathrm{S}$ phase with corresponding changes in chloride channel protein expression and function. $\mathrm{K}^{+}$-channels also change co-temperaneously but the signals creating the links are complex involving ion concentration and phosphorylation [1, 2, 6-8].

In relation to membrane $\mathrm{PD}$, it is correspondingly observed that in G0, PD is very negative (yet chloride does not move out—see above-suggesting tight regulation) but becomes much less negative in proliferative phases (and cancer cell lines). The movement of protons/hydroxyl ions across lipid bilayers is also involved. Thereby, cytosolic
$\mathrm{pH}$ changes during these phase transitions become more alkaline in G1/S transition, for example. The processes interlinked with this decrease in intracellular $\mathrm{pH}$ (hydroxyl exit or proton entry) correlate with a rise in intracellular calcium and changes in $\mathrm{pH}$ sensing mediated by the calcium and phospholipid binding annexin family [7]. Others find regulatory and binding links between annexins and the function of chloride channels such as CFTR [8].

Membrane proteins, cancer, cystic fibrosis and CK2 function

A focus is now placed on a proposed latent role for the cystic fibrosis transmembrane conductance regulator (CFTR) and CK2 with a cation channel, ENaC (Epithelial $\mathrm{Na}^{+}$Channel) as an example of an ion channel cancer link. Without treatment, $\sim 90 \%$ of cystic fibrosis $(\mathrm{CF})$ babies die within the first 2 years of life with uncontrolled exocrine pancreatic failure, a consequent inability to gain weight whilst developing pneumonia with unusual gram positive and gram negative organisms. CF is a mucosal-damaging, autosomal recessive disorder [9] resulting from many mutations in CFTR (ABCC7), an apically located and largely epithelial member of the ABC (ATP-Binding Cassette) superfamily of proteins (but with significant low level expression outside epithelia, including, variably cancer cell lines such as Hela cells). Wild-type CFTR normally contains 1,480 amino acids but alternative splice forms are known, particularly in fetal life, when CFTR is most heavily expressed and especially during rapid epithelial cell proliferation [10]. CFTR is also expressed in red cells, macrophages, bone, etc., but generally, its expression levels in adult tissue are very low or unknown and its functions are relatively obscure outside epithelia [11]. One copy of the defective CFTR gene is carried by as many as 1 in 17 Irish and 1 in 25-50 apparently healthy, predominantly white Europeans suggesting some ancient biological advantage [9]. However, carriage of two CFTR mutations prematurely terminates the lives of 1 in every 2,000-4,000 young adults of European descent (but CF is much rarer in the Far East and almost unknown in Japan $\sim 1$ in a million births; recently reviewed by Walters and Mehta [9]).

Today in the developed world, such is the improvement in childhood nutrition and potent drug treatment for CFinduced gut and lung disease that $\mathrm{CF}$ adults almost outnumber CF children (see report in www.cystic-fibrosis. org.uk, accessed 05/08/2008). In teenage and older CF patients (typically $30-50$ years of age), this disease is associated with an excess incidence of cancer [12]. The data suggest that the most significant association is for gut cancer (5-13-fold excess relative risk in the third and fourth decades of $\mathrm{CF}$ life). Yet, it has to be remembered that such a CF patient has had life-long inflammation in 
both the lung and gut and yet the greatest cancer risk is in the gut epithelium, even in childhood [12]. The reasons are unknown but the CF gut (unlike the lung) is exposed to high fat and exogenously administered pancreatic enzymes. The gut phenotype in CF begins in early fetal life but is of variable severity involving antenatal gut atresia only in about $15 \%$ of CF neonates [9]. It may be that the fetal gut is intrinsically abnormal (i.e. even without atresia) as Ferec and co-workers [13] report a period in early CF fetal life when CFTR expression is undetectable for about three weeks for the commonest form of CF. The significance is unknown. But it is clear that the maximum expression is at around 16-24 weeks in the developing lung which shares lineage with the gut [13]. This notion of intrinsic abnormality is consistent with the findings of Gallagher and Gottlieb who report an abnormally high gut epithelial proliferation rate when cystic fibrosis (CF) mice are null for the murine $\mathrm{CF}$ gene product [14]. This concept of a role for CFTR in fetal gut cell proliferation has received independent support [15]. As CF patients become older (survivors in their sixties are not uncommon), it may be important to monitor their lung cancer risk.

\section{$\mathrm{ABC}$ proteins, CFTR and CK2}

In patch-clamp studies from wild-type epithelial cells, cAMP-elevation (for example, with forskolin) induces protein kinase A (PKA)-activation which immediately stimulates rapid CFTR flickering (passage of chloride current) by enhancing the ratio between open and closed time periods of this channel. This is shown schematically in Fig. 1. But CF cells carrying the common CFTR mutant affecting $70 \%$ of patients (loss of phenylalanine 508,
F508del, 1,479 amino acids; also known as $\Delta$ F508-CFTR) do not respond to such stimuli. The defect in F508delCFTR opening is not due to a defective PKA and yet F508del CFTR only transiently flickers to conduct ions after cAMP elevation but then promptly closes, remaining closed for prolonged periods. Briefly, but detailed elsewhere $[16,17]$ and in Fig. 1,

- When opened, wild-type CFTR will conduct chloride in either an apical-to-inside cell or the reverse direction (depending on the balance of membrane potential and chloride concentration).

- $\mathrm{ABC}$ proteins typically have two nucleotide binding domains (NBD1 and NBD2) which dimerise, ATPdependently, in a ' 69 ' head to tail manner. A dimer is required to open CFTR. Thereafter, ATP hydrolysis drives a cycle of CFTR opening and closing by complex means [17].

- After removal of its water shell, desiccated chloride moves passively through the channel pore deep inside CFTR in a direction driven by the direction of the transmembrane electrochemical driving force field (felt only near the membrane lipids). The direction of motion in to out or vice versa is set by the mostly negative inside membrane potential (thus chloride and other CFTR-permeant anions such as bicarbonate are repelled from the cytosol) and may promote chloride exit from a cell with an open CFTR. Why is the word 'may' used? Remarkably, after cAMP elevation and PKA activation, this net chloride movement from the cell (out of the apical membrane and into the bathing milieu) can occur despite the fact that the intracellular chloride is only $\sim 40 \mathrm{mM}$ and chloride in the fluid
Fig. 1 CK2 inhibition only closes cell attached CFTR. CK2 docks with CFTR using part of a sequence KENIIFGVSYDE on the periphery of the nucleotide binding domain 1 (NBD) that contains F508 whose deletion causes most $\mathrm{CF}$ disease. In the presence of identical concentrations of TBB, a CK2 inhibitor, only the cell attached CFTR shows rapid channel closure (less than $80 \mathrm{~s}$ ) as depicted in the cartoon. If TBB is present PKA cannot open CFTR. Once a patch of membrane is pulled away from the cell (not shown), TBB is without inhibitory effect suggesting the CFTR environment is important for the CK2 inhibition. The details can be found in Pagano et al. [16]
CFTR must have its normal associated proteins for TBB to inhibit channel function CK2 inhibited, CFTR closes CK2 active, CFTR can open
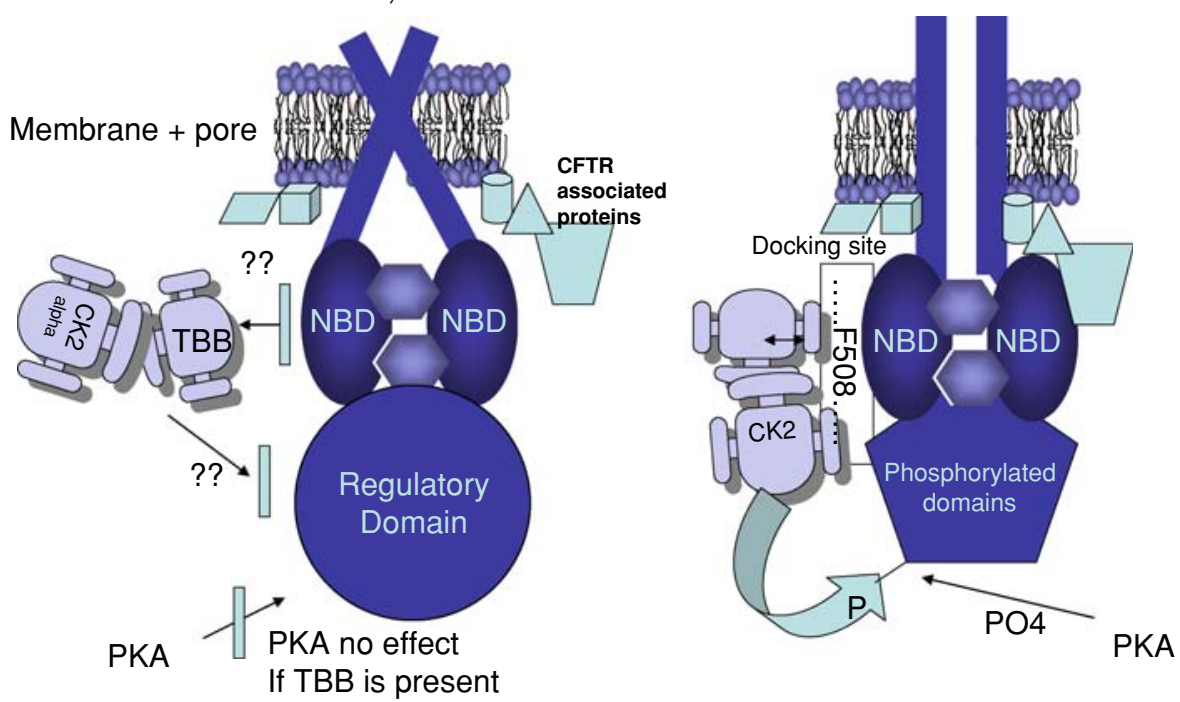
bathing the outer leaflet of the apical membrane is 2.5 times higher at $\sim 100 \mathrm{mM}$. Thus controlling the magnitude of a negative membrane potential is much more important than concentration difference when ions are moved. It follows that controlling a potassium channel can control a chloride channel. If negative enough, this voltage difference (PD) can drive chloride 'uphill' against concentration provided the PD is 'negative enough' (the terms hypo/hyperpolarised are often used as shorthand to refer to transmembrane voltage changes relative to baseline negativity; hyper = more negative inside; reversal potential refers to the balance point between concentration-drive counteracting voltage: i.e. leading to zero net current through a channel, Fig. 1). Similar considerations apply when chloride moves into vesicles inside cells when a proton moves in concert with chloride (not shown).

- Given that CK2 is now thought to control two other $\mathrm{ABC}$ proteins, the cellular cholesterol level regulator ABCA1 [18] and the multi-drug resistance drug efflux pump MDR1/P-glycoprotein [19], it might be that CK2 is a latent factor in multiple $\mathrm{ABC}$ functions [20, 21]. For unknown reasons, CK2 inhibits ABCA1 (preserving cell cholesterol), whereas CK2 activity is permissive for both MDR1 and CFTR. A cellular imbalance of cholesterol accumulation has been proposed in CF epithelial cells [22]. This notion of a wider role for $\mathrm{CK} 2$ in $\mathrm{ABC}$ function is supported by a third $\mathrm{ABC}$ protein recently reported to be controlled by $\mathrm{CK} 2$, albeit one involved in translation at the ribosome [20]. Others have reported an inverse expression relationship between CFTR and P-glycoprotein such that when one is high the other is low in epithelia (and vice versa) [21]. The (unknown) mechanisms of reciprocal expression are mirrored for the epithelial sodium channel (ENaC) controlled by CFTR as described below.

In wild-type cells, CFTR controls ion channels unrelated to CFTR

Non-CFTR channels are also disordered either after the loss of F508 or the whole protein in CFTR null cells. These other affected proteins include anion channels and one cation channel that is highly selective for sodium ions. The latter relationship has been summarised recently by Rotin and co-workers [23]. This, the best studied interaction of CFTR, which nevertheless is unexplained, is between some forms of mutant CFTR and the consequent elevated ENaC activity. When wild-type (but not F508del) CFTR is expressed, ENaC is stabilised at the plasma membrane. Normally ENaC is rapidly internalised in minutes (whereas CFTR takes hours to recycle). Recently, the function and trafficking of this critical CFTR-regulated epithelial sodium channel was also found to be controlled by CK2 [24]. Why should this be relevant?

\section{Speculation}

CK2 is reported to contain an allosteric site with a single chloride ion in its structure that lies at a critical domain in conjunction with fixed water molecules and an inhibitor moiety [25]. It has long been recognised that intracellular chloride concentration regulates $\mathrm{ENaC}$ activity and that extracellular chloride also controls CFTR [reviewed in 26, 27]. Further, we have reported that a bona fide apical membrane-associated CK2 target, nucleoside diphosphate kinase (NDPK), a cancer-related protein manifests differential chloride/anion regulated histidine phosphorylation $[26,27]$ but only when resident within its intact membrane environment.

\section{Discussion}

What may be wrong in CF cells such that cancer can occur?

From an apical membrane and cellular epithelial perspective, the need to maintain the ability to secrete and absorb fluid is crucial. For example, all epithelia are bathed by a liquid elaborated by the epithelium itself and its underlying submucosal glands [26, 27]. This function goes awry in CF partly because the relative activities of CFTR and ENaC become unbalanced (the glands block with mucus). Normally, the negative inside membrane potential should tend to drive chloride out but the very same PD attracts sodium in the opposite direction - into the epithelial cell (external sodium $\sim 130 \mathrm{mM}$, internal $\sim 10-20 \mathrm{mM}$, Fig. 2). The cell expends as much as $40 \%$ of its energy maintaining an intracellular sodium concentration below $20 \mathrm{mM}$ and a CF cell may use even more because sodium channels are overactive making sodium extrusion even more energy utilising. Normally, the apical membrane contains $\mathrm{ENaC}$ (in colon, lung, sweat duct, etc.) and these channels are subject to tight multi-faceted regulation which changes differentially when CFTR is present or mutant. In the absence of CFTR at the apical membrane, ENaC subunits are subject to phosphorylation, ubiquitination, proteolysis and endocytosis keeping its residence time very short [24]. All or some of these posttranslational modifiers change when CFTR is present. They change once again when CFTR is mutant. Perhaps mutant CFTR has an ENaC that is resident for too long. Critically, $\mathrm{ENaC}$ rapidly disappears from the plasma membrane by clathrin-mediated endocytosis but this retrieval of surface exposed apical ENaC is inhibited by the presence of CFTR 
Fig. 2 CK2 stimulates sodium channels by attenuating membrane cycling.

Ubiquitination that normally removes this sodium channel from the cell membrane cannot do so when CK2 prevents the ubiqitin ligase E3 from acting on its normal binding sites located on the regulatory subunits (not shown in detail) because the channel is now phosphorylated close by (see Ref. [24]). The exact relationship between CFTR and $\mathrm{ENaC}$ remains unknown but both are in the same apical epithelial membrane and are separately regulated by the same kinase (CK2) and both are controlled by feedback from ion concentrations such as chloride and sodium inside the cell (not shown)

\section{CK2 targets both CFTR and ENaC by an unknown relationship}

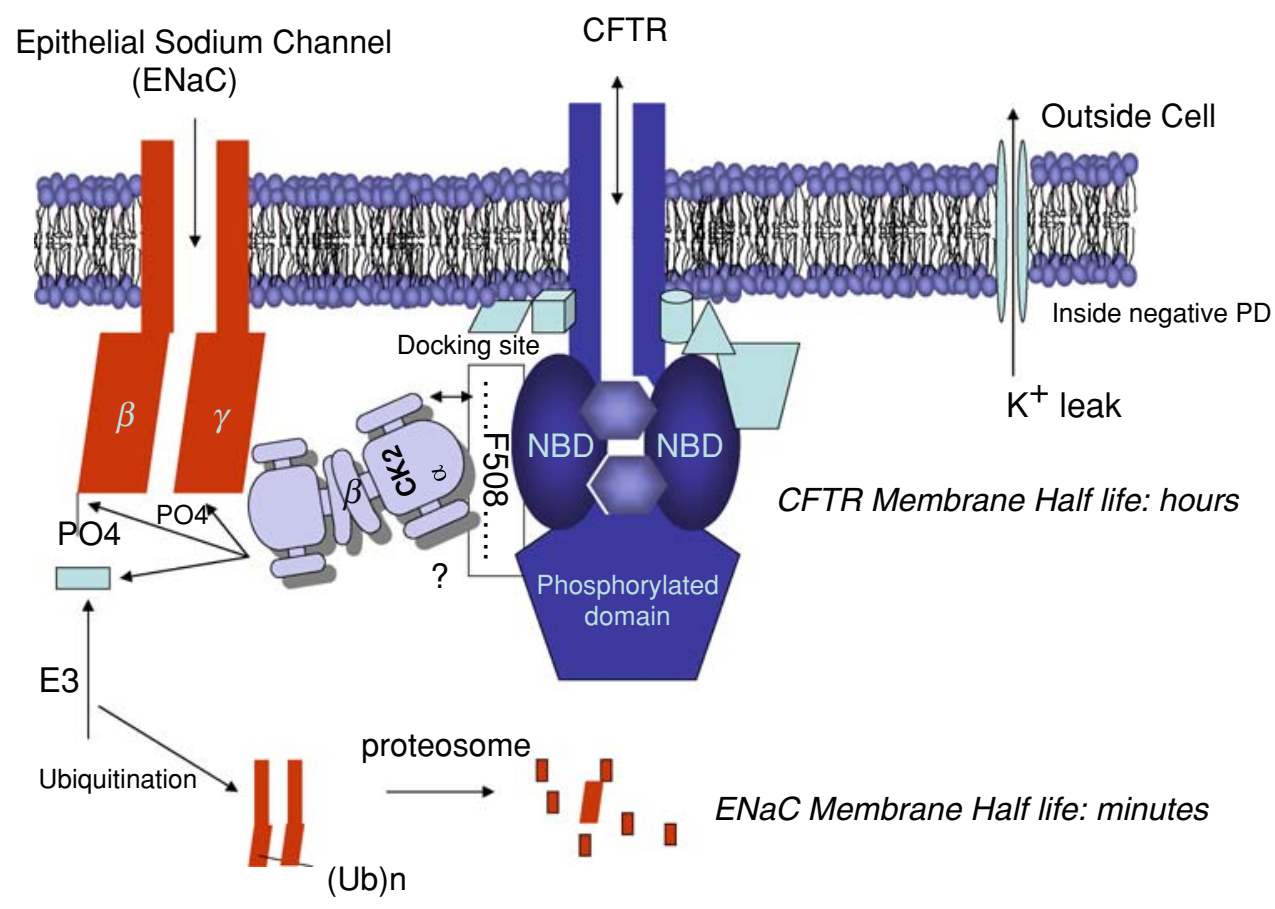

[23]. Separately, Banting and co-workers [28] stress the role of $\mathrm{CK} 2$ (which binds both $\mathrm{ENaC}$ and CFTR, Fig. 2) in coating and uncoating of such clathrin bearing vesicles by a selective interaction only with inositol lipids of certain diphosphate containing lipid classes. CK2 is abundant Xenopus oocytes and yet, strikingly, Kunzelmann and colleagues [24] find that CK2 is not detectable by immunofluorescence in the oocyte membrane when no $\mathrm{ENaC}$ is present. Yet further, when $\mathrm{ENaC}$ is expressed, CK2 $\alpha$ is now easily found in the oocyte membrane. Conversely, CK2 is undetectable in the oocyte membrane when a mutant $\mathrm{ENaC}$ is expressed devoid of its two recognised and functionally important CK2 phosphorylation sites [24]. This suggests that CK2 is carried by the $\mathrm{ENaC}$ to the membrane. In that paper [24] it is also reported that the consensus sites of CK2 phosphorylation of $\mathrm{ENaC}$ lie close to its known sites important for the interaction of two of its subunits with an E3 ubiquitin ligase (Nedd4-2) that normally degrades the protein. The model is shown in Fig. 2. Namely, CK2-dependent phosphorylation of the cytoplasmic loops of the channel prevented access to ubiquitination and thereby delayed its internalisation. Our recent data on CK2 and CFTR also support a model of carriage of $\mathrm{CK} 2 \alpha$ to the membrane by other proteins as has recently been proposed for the kinase scaffold of RAS (KSR) [29]. With respect to Ras/KSR, the assembly of this scaffold (that proximates RAF MEK and ERK) is also CK2 dependent [29] and once again, CK2 is carried to the membrane on cytosolic KSR after cell stimulation.
Thus I propose that CK2 can be 'carried' to many processes in membranes and is permissive (or inhibitory) towards them, presumably after phosphorylation of either itself, its target or both. The mechanisms involved form the focus of our current work and we believe that the future is bright with anticipation that the twin enigmas of excess childhood/young adult CF cancer and CK2 biology may actually provide insight into a common theme, each informing the other. It has always been my belief that detailed study of CF will tell us more about other processes $[30,31]$ that overlap with $\mathrm{CF}$, and CF-related cancer seems a good model to study, particularly with respect to CK2 and the relationship between the fetal and adult gut. The approach taken by Edelman and Balch [30,31] represents the future. They posit that CFTR is a hub of interactions in an unknown pathway(s). In this view CFTR, currently proposed to have only a channel function, may have an alter ego. This review speculates that the enigmatic CK2 on the half century of its discovery can interact with CFTR. This interaction is such that CK2 is controlled differently by CFTR when F508 is deleted [16]. Therefore, this CFTR region, which is accessible on the periphery of the first nucleotide binding domain, controls a cancer-inducing kinase with over 300 reported targets in a CF disease relevant manner. We should not be surprised because after all, in terms of the 'investigational age' of CFTR, it is a mere novice, yet to reach its twentieth year in the human consciousness. 
Acknowledgements The author's laboratory is supported by the Wellcome Trust and Cystic Fibrosis Trust. The views expressed are his own. The author wishes to thank Dr Hongyu Li (University of Bristol) for help with the figures.

\section{References}

1. Suh KS, Yuspa SH (2005) Intracellular chloride channels: critical mediators of cell viability and potential targets for cancer therapy. Curr Pharm Des 11:2753-2764. doi:10.2174/ 1381612054546806

2. Kunzelmann K (2005) Ion channels and cancer. J Membr Biol 205:159-173. doi:10.1007/s00232-005-0781-4

3. Spitzner M, Ousingsawat J, Scheidt K, Kunzelmann K, Schreiber R (2007) Voltage-gated K+ channels support proliferation of colonic carcinoma cells. FASEB J 21:35-44. doi:10.1096/fj.066200com

4. Lee EL, Shimizu T, Ise T et al (2007) Impaired activity of volume-sensitive $\mathrm{Cl}^{-}$channel is involved in cisplatin resistance of cancer cells. J Cell Physiol 211:513-521. doi:10.1002/jcp. 20961

5. Vázquez E, Nobles M, Valverde MA (2001) Defective regulatory volume decrease in human cystic fibrosis tracheal cells because of altered regulation of intermediate conductance $\mathrm{Ca}^{2+}$-dependent potassium channels. Proc Natl Acad Sci USA 98:53295334. doi:10.1073/pnas.091096498

6. Nouri-Sorkhabi MH, Chapman BE, O'Loughlin EV, Li Z, Kuchel PW, Gaskin KJ (2005) NMR measurements of the diffusional permeability of water in cultured colonic epithelial cancer cells. Cell Biol Int 29:441-448. doi:10.1016/j.cellbi.2005.01.006

7. Monastyrskaya K, Tschumi F, Babiychuck EB, Stroka D, Draeger A (2008) Annexins sense intracellular $\mathrm{pH}$ changes during hypoxia. Biochem J 409:65-75. doi:10.1042/BJ20071116

8. Borthwick LA, Mcgaw J, Conner G et al (2007) The formation of the cAMP/protein kinase A-dependent annexin 2-S100A10 complex with cystic fibrosis conductance regulator protein (CFTR) regulates CFTR channel function. Mol Biol Cell 9:33883397. doi:10.1091/mbc.E07-02-0126

9. Walters S, Mehta A (2007) Epidemiology of cystic fibrosis. In: Hodson M, Geddes D, Bush A (eds) Cystic fibrosis, 3rd edn. Hodder Arnold, London

10. Trezise AEO (2007) Exquisite and multilevel regulation of CFTR expression. In: Bush et al (eds) Cystic fibrosis in the 21st century, vol 34, Prog Respir Res. Karger, Basel, pp 11-20

11. Decherf G, Bouyer G, Egée S, Thomas SLY (2007) Chloride channels in normal and cystic fibrosis human erythrocyte membrane. Blood Cells Mol Dis 39:24-34. doi:10.1016/j.bcmd.2007. 02.014

12. Ibele AR, Koplin SA, Bruce L, Slaughenhoupt JV, Kryger AF, Lund DP (2007) Colonic adenocarcinoma in a 13-year-old with cystic fibrosis. J Ped Surg 42:1-3

13. Marcorelles P, Tristan M, Gillet D, Lagarde N, Ferec C (2007) Evolution of CFTR protein distribution in lung tissue from normal and CF human fetuses. Pediatr Pulmonol 42:1032-1040. doi: 10.1002/ppul.20690

14. Gallagher AM, Gottlieb RA (2001) Proliferation, not apoptosis, alters epithelial cell migration in small intestine of CFTR null mice. Am J Physiol 281:G681-G687
15. Umar S, Scott J, Sellin JH, Dubinsky WP, Morris AP (2000) Murine colonic mucosa hyperproliferation. I. Elevated CFTR expression and enhanced cAMP-dependent chloride secretion. Am J Physiol 278:G753-G764

16. Pagano M, Arrigoni G, Marin O (2008) Modulation of protein kinase CK2 activity by fragments of CFTR encompassing F508 may reflect functional links with cystic fibrosis pathogenesis. Biochem (in press)

17. Csanady L, Nairn AC, Gadsby DC (2006) Thermodynamics of CFTR channel gating: a spreading conformational change initiates an irreversible gating cycle. J Gen Physiol 128:523-533. doi: 10.1085/jgp. 200609558

18. Stein Roosbeek S, Frank Peelman F, Annick Verhee A et al (2004) Phosphorylation by protein kinase CK2 modulates the activity of the ATP binding cassette A1 transporter. J Biol Chem 279:37779-37788. doi:10.1074/jbc.M401821200

19. Di Maira G, Brustolon F, Bertacchini J et al (2007) Pharmacological inhibition of protein kinase CK2 reverts the multidrug resistance phenotype of a CEM cell line characterized by high CK2 level. Oncogene 26:6915-6926. doi:10.1038/sj.onc.1210495

20. Paytubi S, Morrice NA, Boudeau J, Proud CJ (2008) The Nterminal region of $\mathrm{ABC} 50$ interacts with eukaryotic initiation factor eIF2 and is a target for regulatory phosphorylation by CK2. Biochem J 409:223-231. doi:10.1042/BJ20070811

21. Maitra R, Hamilton J (2005) Arsenite regulates cystic fibrosis transmembrane conductance regulator and P-glycoprotein: evidence of pathway independence. Cell Physiol Biochem 16:1-3. doi:10.1159/000087737

22. Jiang D, Fang D, Kelley TJ, Burgess JD (2008) Electrochemical analysis of cell plasma membrane cholesterol at the airway surface of mouse trachea. Anal Chem 80:1235-1239. doi:10.1021/ ac7019909

23. Lu C, Jiang C, Pribanic S, Rotin D (2007) CFTR stabilizes ENaC at the plasma membrane. J Cyst Fibros 6:419-422. doi:10.1016/j. jcf.2007.03.001

24. Bachhuber TA, Almaca J, Aldehini J et al (2008) Regulation of the Epithelial $\mathrm{Na}^{+}$channel by protein kinase CK2. J Biol Chem 283:13225-13232

25. Raaf J, Brunstein E, Issinger OG, Niefind K (2007) The CK2a/ $\mathrm{CK} 2 \mathrm{~b}$ interface of human protein kinase CK2 harbors a binding pocket for small molecules. Chem Biol 15:111-117. doi: 10.1016/j.chembiol.2007.12.012

26. Mehta A (2007) The cystic fibrosis transmembrane recruiter the alter ego of CFTR as a multi-kinase anchor. Pflugers Arch 455:215-221. doi:10.1007/s00424-007-0290-7

27. Mehta A (2005) CFTR: more than just a chloride channel. Pediatr Pulmonol 39:292-298. doi:10.1002/ppul.20147

28. Korolchuk VI, Cozier G, Banting G (2005) Regulation of CK2 activity by phosphatidylinositol phosphates. J Biol Chem 280:40796-40801. doi:10.1074/jbc.M508988200

29. Ritt CD, Zhou M, Conrads T et al (2007) CK2 is a component of the KSR1 scaffold complex that contributes to Raf kinase activation. Curr Biol 17(2):179-184. doi:10.1016/j.cub.2006.11.061

30. Ollero M, Brouillard F, Edelman A (2006) Cystic fibrosis enters the proteomics scene: new answers to old questions. Proteomics 6(14):4084-4099. doi:10.1002/pmic.200600028

31. Moyer BD, Balch WE (2001) A new frontier in pharmacology: the endoplasmic reticulum as a regulated export pathway in health and disease. Expert Opin Ther Targets 5(2):165-176 